\title{
Significance of tumor self-seeding as an augmentation to the classic metastasis paradigm
}

\section{Philip Hahnfeldt}

Center of Cancer Systems Biology, St Elizabeth's Medical Center, Tufts University School of Medicine, 736 Cambridge Street, CBR-175, Boston, MA 02135, USA = Tel.: +1 6177892998 = Fax: +1 6175627142 - philip.hahnfeldt@tufts.edu

Evaluation of: Kim M-Y, Oskarsson T, Acharyya S et al.: Tumor self-seeding by circulating cancer cells. Cell 139, 1315-1326 (2009). Augmenting our understanding that metastasis involves the spread of tumor cells to distant sites, this study describes an overlooked phenomenon - the seeding of metastases to the primary tumor itself. An aggressive population of circulating tumor cells (CTCs) within the primary tumor, which has already surmounted the challenge of entering and exiting the circulation, is revealed. The self-seeding principle was demonstrated using mice implanted with both 'recipient' unlabeled nonaggressive tumors and with homotypic 'donor' tumors, consisting of green fluorescent protein-labeled metastatic variants. When the donor tumor implants were instead nonaggressive, labeled cells isolated from the recipient tumors also exhibited strong self-seeding potential when re-implanted as donors, demonstrating selection for and amplification of a metastatic phenotype. The isolated CTC population possessed gene classifiers for all typical metastatic sites (bone, lung and brain). Metastatic and indolent populations could both chemoattract metastatic cells, while the CTCs demonstrated infiltrative capacity. CTCs were found to enhance primary tumor growth, probably through stromal paracrine effects. A self-seeding mechanism, driven by tumor chemoattraction and CTC infiltration, is concluded to play a critical role in tumor malignancy.

\section{Summary of methods \& results}

Expanding on the conventional thinking that metastasis entails dissemination of tumor cells to distant sites, Kim et al. describe how tumors may be seeded by their own metastases [1] To demonstrate the process, the authors first used an MDA-MB-231 (MDA231) human breast tumor line for which a metastatic subpopulation (MDA231-LM2) had already been characterized. They implanted green fluorescent protein-labeled metastatic cells into one mammary gland as the 'donor' tumor and either the metastatic or more indolent parental cells into a contralateral gland as the 'recipient' tumor. After 60 days, labeled cells from the donor tumor appeared as small patches in $85 \%$ of recipient tumors. Results were generalizable to metastatic donor breast tumors and parental-line recipients; to the metastatic donor colon tumor SW630-LM1 and its less aggressive recipient counterpart SW630; and to the syngeneic donor breast tumor 4T1 (metastatic) and its recipient $67 \mathrm{NR}$ (poorly metastatic) in immunocompetent mice. To demonstrate that mammary tumors could also be seeded by metastases in vivo, lung colonies were generated from labeled MDA231-LM2 injected by tail vein and mammary tumors were then implanted.
Seeding of the mammary tumors with cells shed from the lung colonies was observed in ten out of 11 mice.

The less aggressive, parental human lines SW620 (colon), A375 (melanoma) and MDA231 were relatively ineffectual as donor populations, showing eight- to 35 -fold less seeding ability than their metastatic counterparts. To characterize the cells that managed to seed from these less aggressive lines, contralateral glands or flanks were injected with green fluorescent protein-labeled donor cells and with unlabeled recipient cells from either the parental MDA231 or the A375 line. After 50 days, seeded tumors were evident. The seeded tumors were removed and sorted by FACS to isolate the labeled cell populations MDA231-S1a, MDA231-S1b and A375-S1. When reimplanted as donor tumors, MDA231-S1a and -S1b were found to have more than 100-times the seeding ability of the parent cells. When injected into the circulation (intracardially), MDA231-S1a and A375-S1 cells had six-times the seeding efficiency of parental cells.

Focusing now on the parent MDA231 line and its metastatic derivatives, a comparison of transcriptional profiles showed 72 genes to be upregulated more than three-times in the MDA231-S1a and -S1b subpopulations versus
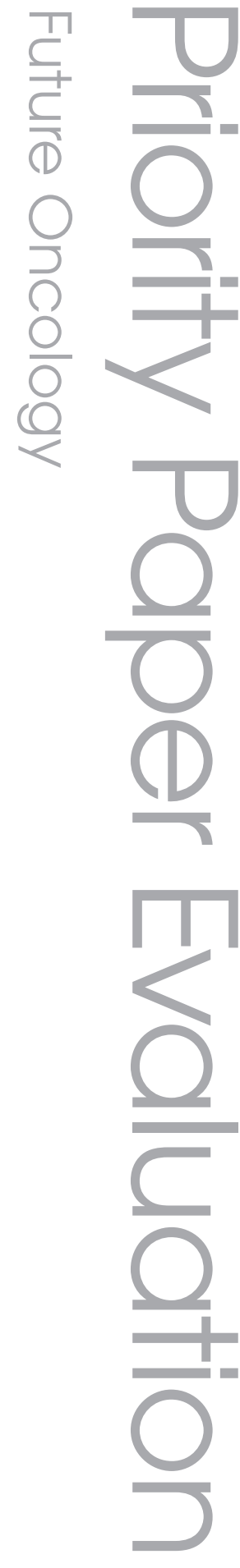

\section{Keywords}

- chemoattraction

- circulating tumor cell hypervascularization

- indolent tumor $=$ infiltration

- invasiveness - metastasis

- stromal paracrine effect

- tumor cell selection

- tumor stem cell

\section{future icost fsg}


the parent. Genes associated with bone, brain and lung metastases were included. Transcriptomes also showed heightened expression of bone metastasis, lung metastasis and brain metastasis gene expression classifiers previously derived from human breast cancer cells metastatic to these organs [2-5]. This was mirrored by the increased ability of S1a cells to populate the bone, lungs and brain.

To define potential chemoattractant and infiltration functions, labeled MDA231 or MDA231-LM2 cells were injected intracardially into mice previously implanted with MDA231 tumors. By day 7, labeled cells were almost exclusively localized to the implanted tumors (but not control glands). Again, the MDA231-LM2 variants seeded more effectively than the parental cells. Analogously, metastatic A375-BoM2 cells seeded better than the parental A375 cells to implanted A375 tumors. The putative attractant and infiltration processes were examined more directly using a transendothelial migration assay. MDA231-LM2 and A375-BoM2 cells migrated more effectively through endothelial cell layers towards media conditioned with MDA231 (or MDA231-LM2) and A375 (or A375-BoM2) cells, than towards media conditioned with the normal cell counterparts, MCF10A (breast epithelial cells) and $\mathrm{HaCat}$ (keratinocytes), respectively. The media of MDA231 and MDA231-LM2 cells were equivalent as attracting sources, in line with the results from the recipient tumor experiments. Parental MDA231 cells showed little transmigration ability. The results showed that cancer cells are generally chemoattractive for their own cell types and that their more aggressive counterparts are more efficient responders to the chemoattractant.

Tracking down the cytokines responsible, the investigators found IL- 6 and IL- 8 to be more highly expressed in MDA231 and MDA231-LM2 than in MCF10A and IL-8 more highly expressed in A375-BoM2 than in HaCat. When IL-6 and IL-8 were added to the media of the normal cell counterparts, MCF10A and HaCat, respectively, transwell migration of MDA-231-LM2(-S1a) and A375BoM2 cells was stimulated and was sustained or even amplified by the presence of an endothelial layer. Examining IL-6 further with respect to MDA231 cells, knockdown of the IL-6 receptor lowered the self-seeding capacity of MDA231-S1a cells. These data together argue that IL- 6 and IL- 8 from MDA231 and A375 tumors serve as chemoattractors for their metastatic counterparts.
In a similar search for candidate infiltration genes expressed in the circulating tumor cells (CTCs), matrix metalloproteinase 1 (MMP1), Fascin1 (FSCN1; an actin 1 crosslinking protein implicated in invasiveness) and CXCL1 (a mediator of leukocyte recruitment) stood out as being associated with seeding to the lungs and brain $[2,4]$ and to the lung metastasis classifier signature for lung relapse [4]. These were expressed at levels 11- to 40-fold higher in the MDA231S1a and Slb populations than in the parental cells. In transendothelial migration assays, silencing of $M M P 1$ alone had no effect, but in combination with FSCN1 (or when FSCN1 was silenced alone), potent responses were observed. These data show that MMP1 and FSCN1 may mediate infiltration.

To establish whether self-seeding can affect tumor growth, MDA231 tumors were implanted, followed 10 days later by intracardiac injection of MDA231-LM2 cells. Tumor growth was accelerated in a manner well beyond what could be accounted for by either population alone, and even exceeded that observed for the MDA231S1a and-S1b seeder populations. It was concluded that the MDA231-LM2 cells enhance the growth of MDA231 tumors through a stromal interaction.

Infiltrated tumors also contained more vasculature and greater infiltration by leukocytes, as assessed by CD31 and CD45, respectively. Results were recapitulated for the A375 cells and with $4 \mathrm{~T} 1$ cells in a syngeneic mouse model. Although CXCL1 did not affect transendothelial migration, mediation of leukocyte recruitment by CXCL1 proved to be a contributor to stromal recruitment. Knockdown of CXCL1 in MDA231-S1a cells reduced leukocyte recruitment into MDA231 tumors. The results are consistent with the proposed growth-stimulating effect of cell seeding through effects on the surrounding stroma.

\section{Discussion}

In the context of tumor metastasis, the prospect of tumor cells re-invading their source tumor is a plausible extension to our mechanistic thinking. Indeed, the primary tumor may be easier than a distant organ to infiltrate, having already paved the way for tumor cell entry with leaky vasculature. Moreover, the local adaptation of the primary, coupled with the invasive competence of returning metastatic cells, would make 'self-seeding' an important contributor to tumor aggressiveness. Ample evidence for the possibility has been presented. In breast, 
colon and melanoma models, the ability of metastatic implants to seed their more indolent counterpart implants was demonstrated. Direct seeding of implanted tumors from metastatic sites and from injections of metastatic cells into the circulation were also observed. Both metastatic cell lines and seeder cells selected from indolent tumors were similarly efficient at seeding parent tumors and were much more efficient at this than the parent tumor cells in general. These data suggest that seedingcompetent cells, CTCs, pre-exist in the source tumor and that the actions of circulation and re-infiltration are highly selective for these aggressive cells. Selected cells bear gene signatures resembling bone, brain and lung metastatic populations, the mixture perhaps reflecting the invasive competence shared by these organ-specific subtypes.

However, no seeding was observed in control orthotopic mammary glands, suggesting an attractive role for the parent tumor. The cytokines IL-6 and IL-8 were implicated for the tumor lines studied, based on the failure of transendothelial migration in knockdown studies. This accords with other findings tying one or both of these cytokines to chemoattraction, tumor load and poor prognosis. In order to explain the ability of CTCs to re-infiltrate the source tumor upon arrival, a second set of genes was considered. Among the 'infiltrative' genes flagged by transwell migration assay were $M M P 1$ and $F S C N 1$, genes known to be involved in the relapse of certain tumors. A two-step process is suggested - first, chemoattracting signals produced by the tumor or immune cells at the primary site act to draw the CTCs, then signals produced by the attracted cells facilitate infiltration of the primary. Upon arrival of the aggressive CTCs to the primary, an interactive process heightens the tumor growth rate. Consistent with other experimental observations of metastasis, responses include increased angiogenesis and immune cell recruitment [6]. None of these can be explained by tumor cell populations alone (the CTCs and parent cells have comparable growth rates), pointing to paracrine stromal signaling as being contributory. It is concluded that attributes of metastatic cells may be visited upon and amplified in the primary, explaining, for example, hypervascularization and the link between larger tumors and poorer prognoses. A study of metastasis might therefore benefit, curiously, from a closer look at the primary.

\section{Future perspective}

Since Paget first proposed his 'seed-and-soil' hypothesis for metastatic spread in 1889 [7], the notion that tumors grow in favored environmental conditions has attained broad acceptance. The idea that one such location might be the original tumor site itself, then, is eminently logical - some might say 'obvious'. However, this belies the shrewdness of the observation. The concept of self-seeding reported by Kim et al. both reconciles standing issues and raises fascinating new ones [1].

Since the tumor inherits certain characteristics from its returning metastases, heightened aggressiveness may be explained by both existing metastases and by the potentiation of further metastases in the primary. At the same time, as the authors point out, the process appears to be selective rather than adaptive. This means the primary already has the potential to generate seeders without the need for an adaptive metastatic experience. If true, the act of circulating serves only to concentrate and expand existing cell types most fit to survive the excursion. Evidence for this task-driven dynamic was observed by Shipitsin et al. [8]. They found a higher number of CD $24^{+}$cells in distant metastases, despite the fact that tumors that produce the metastases (mainly to bone) had a higher fraction of CD24- cells [9]. In this way, the stem-like CD $44^{+} / \mathrm{CD} 24^{-}$status thought to be necessary for breast metastasis formation gave way to a more differentiated cell composition once the formal task of metastasis was completed.

This suggests the original 'nonaggressive' tumor has, in fact, an immense strategic repertoire, with tailored aggressive responses awaiting the proper selective challenges. This prospect raises other selection-based scenarios for tumor development. Our group previously described a local version of the self-seeding phenomenon that we termed 'self-metastasis' [10]. In an in silico model of tumor growth, space constraints were seen to act as selective influences on a dynamical interaction between migrating cancer stem and nonstem cells. What we concluded in that study was quite analogous to the present case - tumor growth may be enhanced by a self-seeding process. We found that tumors can expand through local metastatic cluster formation, enabled by the freeing of space owing to cell death and migration of stem cells away from the tumor surface. This model also resolves important issues; for example, how cell death and immunity may 
actually promote tumor growth. It has also found direct clinical relevance to inflammatory breast cancer [11]. Inflammatory breast cancer is a disease characterized by a central growth, with smaller nodules of carcinoma at its periphery and interspersed intravascular emboli. The nodules and emboli are in close association with the main tumor, much as would be predicted from space-enabled local stem cell migration followed by re-assimilation of the resulting clusters. The emboli have also been observed clinically to have a stem-like phenotype [12]. Vermeulen [11] noted the selfmetastasis principle to be a possible realization of the original self-seeding proposition of Norton and Massague [13], which envisioned that some proportion of self-seeds may be either mimicking or synonymous with cancer stem cells [14]. Looking ahead, if indeed the observable responses are limited only by the creativity of the selection process, self-seeding may join a long list of tumor-defining dynamics that have yet to be uncovered.

Financial \& competing interests disclosure
The author has no relevant affiliations or financial
involvement with any organization or entity with a
financial interest in or financial conflict with the sub-
ject matter or materials discussed in the manuscript.
This includes employment, consultancies, honoraria,
stock ownership or options, expert testimony, grants or
patents received or pending, or royalties.
No writing assistance was utilized in the production
of this manuscript.

\section{Executive summary}

\section{Seeding characteristics}

- Cells from metastatic breast tumors seed their co-implanted homotypic parent tumors.

- Metastatic MDA231-LM2 cells seed MDA231-LM2 and parent MDA231 tumors.

- This phenomenon is generalizable to other metastatic-parent combinations (SW630 and 4T1).

- Lung colonies made from tail vein injections of MDA231-LM2 could also seed previously implanted MDA231 tumors.

- Cells from parent MDA231 donors can also seed co-implanted MDA231 tumors, albeit at a low rate. The seeder cells isolated (MDA231-S1a and -S1b) had dramatically heightened seeding ability, similar to the metastatic line MDA231-LM2.

- When the seeder populations were introduced into the circulation, they also seeded previously implanted tumors.

\section{Genes upregulated in seeder populations MDA231-S1a \& -S1b compared with MDA231 parent cells}

- A total of 72 genes associated with bone, lung and brain metastasis and expressions of bone, lung and brain metastasis classifiers were upregulated.

\section{Defining chemoattractant functions}

- Intracardial injections of MDA231 or MDA231-LM2 cells into mice previously implanted with MDA231 recipient tumors recapitulated the seeding responses noted for donor tumors.

- The same was observed for injected A375 and A375-BoM2 cells previously implanted with A375 recipient tumors.

- Transendothelial migration assays of donor cells towards media conditioned with recipient tumors recapitulated the above results. Less migration towards media conditioned with the normal cell counterparts MCF10A (breast epithelial cells) and HaCat (keratinocytes) was observed.

- Parental MDA231 cells were less efficient at transmigration.

- The last two results show that cancer cells generally are chemoattractive for their own cell types and that their more aggressive counterparts are more efficient responders.

\section{Chemoattractant cytokines}

- IL-6 and IL-8 show higher expression in the cancers examined than in normal cell counterparts.

- Migration of MDA-231-LM2(-S1a) and A375-BoM2 towards media conditioned with normal counterparts occurred when IL-6 and IL-8 were added to media.

- Knockdown of the IL-6 receptor lowered the seeding capacity of MDA231-S1a cells.

\section{Candidate infiltration genes}

- MMP1, Fascin-1 (FSCN1) and CXCL1 are associated with seeding to lung and brain and to the lung metastasis signature for relapse.

- These genes are expressed 11- to 40-fold more in MDA231-S1a,b cells than parental cells.

- Transendothelial experiments identified MMP1 and FSCN1 as potential mediators.

\section{Self-seeding affects tumor growth \& development}

- Intracardiac injections of MDA231-LM2 cells accelerated the growth of previously implanted MDA231 tumors.

- Infiltrated tumors showed more vascularity and leukocyte infiltration.

- The results were recapitulated for A375 cells and 4T1 cells in a syngeneic model.

- CXCL1 was identified as a recruiter of leukocyte infiltration. The knockdown of this gene inhibited the response.

- It was concluded that self-seeding stimulates tumor growth by means of effects on the stroma. 


\section{Bibliography}

Papers of special note have been highlighted as:

- of interest

-" of considerable interest

1. Kim M-Y, Oskarsson T, Acharyya S et al: Tumor self-seeding by circulating cancer cells. Cell 139, 1315-1326 (2009).

2. Bos PD, Zhang XH, Nadal C et al.: Genes that mediate breast cancer metastasis to the brain. Nature 459, 1005-1009 (2009).

3. Kang Y, Siegel PM, Shu Wet al.: A multigenic program mediating breast cancer metastasis to bone. Cancer Cell 3, 537-549 (2003).

4. Minn AJ, Gupta GP, Siegel PM et al.: Genes that mediate breast cancer metastasis to lung. Nature 436, 518-524 (2005).

- Background genetic information relating to the phenomenon of chemoattraction and infiltration is presented.

5. Minn AJ, Kang Y, Serganova I et al.: Distinct organ-specific metastatic potential of individual breast cancer cells and primary tumors. J. Clin. Invest. 115, 44-55 (2005).
6. Lorusso G, Rüegg C: The tumor microenvironment and its contribution to tumor evolution toward metastasis. Histochem. Cell Biol. 130, 1091-1103 (2008).

7. Paget $S$ : The distribution fo secondary growths in cancer of the breast. Lancet 1, 571-573 (1889).

8. Shipitsin M. Campbell LL, Argani P et al: Molecular definition of breast tumor heterogeneity. Cancer Cell 11, 259-273 (2007).

9. Abraham BK, Fritz P, McClellan M et al.: Prevalence of CD44 /CD24-/low cells in breast cancer may not be associated with clinical outcome but may favor distant metastasis. Clin. Cancer Res. 11, 1154-1159 (2005).

10. Enderling H, Hlatky L, Hahnfeldt P: Migration rules: tumours are conglomerates of selfmetastases. Br. J. Cancer 100, 1917-1925 (2009).

- Another mechanistic basis for the self-seeding phenomenon is presented, based on local kinetic selection assessed in silico.
11. Vermeulen PB, van Laere SJ, Dirix LY: Inflammatory breast carcinoma as a model of accelerated self-metastatic expansion by intravascular growth. Br. J. Cancer 101, 1028-1029 (2009).

12. Xiao Y, Ye Y, Yearsley K et al.: The lymphovascular embolus of inflammatory breast cancer expresses a stem cell-like phenotype. Am. J. Pathol. 173, 561-574 (2008).

13. Norton L, Massague J: Is cancer a disease of self-seeding? Nat. Med. 12, 875-878 (2006).

- General principles for the self-seeding phenomenon are set forth, expanding on the specific study of circulating self-seeders presented here.

14. Al-Hajj M, Clarke MF: Self-renewal and solid tumor stem cells. Oncogene 23, 7274-7282 (2004).

- Functional concept of the cancer 'stem' cell is reviewed. 